\title{
Sirt3 enhances glioma cell viability by stabilizing Ku70-BAX interaction
}

This article was published in the following Dove Press journal:

OncoTargets and Therapy

\author{
Ke Luo* \\ Wei Huang* \\ Shuang Tang \\ Department of Neurosurgery, Suining \\ Central Hospital, Suining, Sichuan, \\ China \\ *These authors contributed equally \\ to this work
}

Background: As one of the most prevalent malignancies, glioma is characterized by poor prognosis and high mortality rate. Glioma patients may show completely distinct clinical outcomes due to their different molecular patterns. Sirtuin 3 (Sirt3) participates in aging, stress resistance, and metabolic regulation. Here we aimed to test the expression and function of Sirt3 in glioma.

Methods: We enrolled 114 patients and tested the protein level of Sirt3 in their glioma tissues. The correlation between prognosis and Sirt3 was evaluated by univariate and multivariate analyses. We also conducted cellular experiments in U87 and U251 glioma cells, including overexpression and knockdown assays.

Results: Sirt3 expression was lower in glioma tissues than normal brain tissues. Higher Sirt3 is significantly correlated to advanced tumor grade ( $P=0.004)$, showing its potential role in cancer progression. Consistently, univariate and multivariate analyses identified Sirt3 as an independent prognostic factor $(P=0.017)$. Patients with higher Sirt3 expression showed significantly shorter overall survival time. Moreover, overexpression of Sirt3 in either cell line enhanced cell viability, while silencing Sirt3 attenuated cell growth. Molecular assays showed Sirt3 can deacetylate Ku70 protein, therefore stabilizing the Ku70-BAX interaction. Since Ku70 can help prevent BAX transporting into mitochondria and decrease cell apoptosis, Sirt3 protein may play roles in maintaining cell viability. In addition, silencing Ku70 inhibited the pro-proliferative effect by Sirt3.

Conclusion: Taken together, our results not only identified the prognostic role of Sirt3 in glioma patients but also provided signaling pathway evidence for its functioning mechanisms.

Keywords: glioma, prognosis, Sirt3, viability

\section{Introduction}

Primary glioma is the most common type of neoplasm in the central nervous system. ${ }^{1}$ Gliomas are divided into four grades (I-IV) according to the WHO classification. ${ }^{2}$ The treatment methods toward glioma include chemotherapy, radiotherapy, and surgical intervention. ${ }^{3}$ However, the curative effect varies among individuals. Although the patients with WHO grade I-II have a better overall survival, the 5-year survival rate for grade IV patients is less than $5 \%{ }^{4}$ The pathogenesis and progression mechanisms of glioma have drawn great attention in the past decades; however, the overall improvement in patients' outcomes is far from satisfying. ${ }^{5}$ In addition, the current prediction models on patients' prognosis are largely based on the clinicopathological characteristics, while the efficiency varies in different studies. Therefore, a more detailed stratified approach based on molecular expression patterns is still one of the major challenges.

Sirtuins are a class of protein deacetylases and are highly conserved in many species. ${ }^{6}$ Sirtuins consist of seven protein members from sirtuin 1 to sirtuin $7 .{ }^{7}$ Different sirtuin subtypes show completely distinct subcellular localizations and functions.
Correspondence: Shuang Tang Department of Neurosurgery, Suining Central Hospital, \#I27 Desheng West Road, Suining, Sichuan 629000, China Tel +86 I34 I936 2776 Email huocha025588@I63.com BY NG and incorporate the Creative Commons Attribution - Non Commercial (unported, v3.0) License (http://creativecommons.org/licenses/by-nc/3.0/). By accessing the work you
hereby accept the Terms. Non-commercial uses of the work are permitted without any further permission from Dove Medical Press Limited, provided the work is properly attributed. For permission hereby accept the Terms. Non-commercial uses of the work are permitted without any further permission from Dove Medice
for commercial use of this work, please see paragraphs 4.2 and 5 of our Terms (https://www.dovepress.com/terms.php). 
As for sirtuin 3 (Sirt3), it is initially characterized as a mitochondrial deacetylase involved in respiratory chain, tricarboxylic acid cycle, and fatty acid oxidation. ${ }^{8}$ Subsequently, the expression of Sirt3 was also detected in cytoplasm and nucleus with novel substrates. ${ }^{9}$ From the physiological aspect, Sirt3 participates in regulating cardiovascular functions, aging, and metabolic process. ${ }^{10}$ Most recently, Sirt3 was reported to function in tumor progression. ${ }^{11}$ For example, it inhibits tumor progression of breast cancer, hepatocellular carcinoma, and leukemia. ${ }^{12-14}$ However, the role of Sirt3 seems more like tumor promoter in colon cancer, gastric cancer, melanoma, and renal cancer. ${ }^{15-18}$ Therefore, Sirt 3 can play both tumor-suppressing and tumor-promoting roles in different tumor types.

Here we initially investigated the expression and function of Sirt3 in glioma and described the mechanisms by which it modulates glioma progression. Briefly, Sirt3 was identified to be expressed higher in glioma tissues than normal brain tissues, which was significantly correlated to advanced tumor stages. Besides, the higher Sirt3 protein levels indicated poorer overall survival of glioma patients. Cellular experiments were performed in two glioma cell lines; both demonstrated the possible signaling axis of Sirt3-Ku70BAX (Bcl-2-associated X protein) in modulating glioma progression.

\section{Methods}

\section{Patients and samples}

This study was approved by the Ethics Committee of Suining Central Hospital. Written informed consents were obtained from all patients. Formalin-fixed paraffin-embedded glioma tissues were obtained from surgery in 114 randomly selected patients during 2002-2012 in Suining Central Hospital. Another 22 glioma samples, which were fresh frozen in liquid nitrogen, were also collected in our hospital. We also purchased 17 normal brain tissue samples from Cureline (South San Francisco, CA, USA). All patients were followed-up ranging from 8 to 71 months.

\section{Immunohistochemistry (IHC) and IHC evaluation}

As described by others, ${ }^{19}$ IHC was used to evaluate the Sirt3 protein expression in glioma tissues. Briefly, deparaffinized and rehydrated slides were treated in $10 \mathrm{mM}$ citrate buffer ( $\mathrm{pH}$ 6.0) at $95^{\circ} \mathrm{C}$ for antigen recovery, followed by quenching the endogenous peroxidase activity with $3 \% \mathrm{H}_{2} \mathrm{O}_{2}$ incubation. Five percent FBS was used for blocking the nonspecific binding sites. Slides were then incubated with primary antibody (anti-Sirt3; Abcam, Cambridge, MA, USA; \#ab86671;
$1: 200)$ at $4^{\circ} \mathrm{C}$ overnight. DAB staining kit (Tiangen, China) was used to detect the immunoreactivity according to the manufacturer's instructions.

The level of Sirt3 was determined by the degree of staining intensity and the percentage of positively stained cells. Examination and scoring were performed by two pathologists independently. In brief, weak staining, moderate staining, and strong staining were scored as 1, 2, and 3, respectively. The percentages of positively stained cells were scored as follows: 1 for less than $20 \%, 2$ for $20 \%-50 \%$, and 3 for $50 \%-100 \%$. The final IHC score was defined by multiplying the the two scores above, ranging from 1 to 9 . Tissues with a final score no more than 4 were regarded as low-expression cases; otherwise, it will be grouped into the Sirt3 high-expression group.

\section{Quantitative real-time PCR (RT-qPCR)}

Total RNA was extracted from fresh-frozen specimens by using the Trizol reagent (Invitrogen, Carlsbad, CA, USA) following a standard protocol. ${ }^{20}$ Then the RNA was reversely transcribed by using the Primer-Script RT Enzyme Mix. RT-qPCR was carried out using the SYBR Premix Ex Tag (Takara, Japan) according to the instructions. GAPDH was used as normalization control, and the primers were presented as following:

Sirt3-F: 5'-ACCCAGTGGCATTCCAGAC-3'; Sirt3-R: 5'-GGCTTGGGGTTGTGAAAGAAG-3'; GAPDH-F: 5'-AGGGCTGCTTTTAACTCTGGT-3'; GAPDH-R: 5'-CCCCACTTGATTTTGGAGGGA-3'.

\section{Cell culture and transfection}

Normal human astrocytes (NHA cells) were purchased from Lonza (Basel, Switzerland). The human glioma cells (U87 and U251) were obtained from the Cell Bank of the Chinese Academy of Sciences (Shanghai, China). All cells were cultured in DMEM medium supplemented with $10 \%$ FBS (Hyclone, South Logan, UT, USA) and 1\% streptomycin and penicillin in a humidified atmosphere of $5 \% \mathrm{CO}_{2}$ at $37^{\circ} \mathrm{C}$. The Sirt3 siRNA (sequence: CATCCCTACATGCAGATGAAA), Ku70 siRNA (sequence: GAGUGAAGAUGAGUUGACA), Sirt3 overexpressing plasmid construct, and $\mathrm{Ku} 70$ overexpressing plasmid construct used in this study were synthesized by Genechem (Shanghai, China). The transfections were carried out using Lipofectamine 3000 according to the manufacturer's procedure (Invitrogen). ${ }^{21}$

\section{Western blotting}

Cells were scraped with PBS and lysed with ice-cold RIPA lysis buffer (Beyotime Biotechnology, Shanghai, China) 
supplemented with protease inhibitor cocktail. The lysates were centrifuged at $12,000 \times g$ for 15 minutes at $4{ }^{\circ} \mathrm{C}$, and the precipitate was discarded. Equal amount protein $(10 \mu \mathrm{g})$ was electrophoresed with sodium dodecyl sulfate-polyacrylamide gel and then the proteins were transferred to a polyvinylidene difluoride membrane (Merck Millipore, Kenilworth, NJ, USA). The primary antibodies used in this study included anti-Sirt3 (Abcam, \#ab86671), anti-Ku70 (Invitrogen, \#MA513110), anti-acetyl (Abcam, \#ab80178), anti-BAX (Cell Signaling Technology, Danvers, MA, USA; \#2774), anticytochrome c (Cell Signaling Technology, \#4280), anticaspase 3 (Cell Signaling Technology, \#9662), and anti- $\beta$-actin (Cell Signaling Technology, \#3700). After incubation with primary and secondary antibodies, the immunoreactivity bands were visualized by using an enhanced chemiluminescence substrate kit (Thermo Scientific, Pittsburgh, PA, USA). ${ }^{22}$

\section{Immunoprecipitation}

To identify the interaction between Sirt3 and Ku70, we performed antibody-affinity immunoprecipitation in U251 cells transfected with Ku70 plasmid. Briefly, transfected cells were harvested and lysed in lysis buffer. After centrifuged at $12,000 \times g$ at $4^{\circ} \mathrm{C}$ for 15 minutes, supernatants were added with $\mathrm{Ku} 70$-Protein G mixture (Santa Cruz Biotechnology) and incubated overnight at $4^{\circ} \mathrm{C}$. Protein $\mathrm{G}$ was then spinned down and washed with lysis buffer for three times. ${ }^{23}$ Finally, the immunoprecipitated proteins were subjected to Western blot analyses.

\section{Mitochondrial fractionation}

To determine the protein distribution in mitochondria, cultured cells were harvested for mitochondrial fractionation by using a mitochondrial isolation kit for mammalian cells (Thermo Scientific) according to the manufacturer's protocol. $^{24}$

\section{Cell viability assay}

To evaluate the effect of Sirt3 on tumor cell viability, plasmid or siRNA transfected cells were seeded at $2 \times 10^{4}$ cells per well in a 96-well plate and cultured in DMEM. At designated time points, cell viability was assessed by a Cell Counting Kit-8 (Dojindo, Japan) according to the manufacturer's instructions. ${ }^{25}$ Briefly, $10 \mu \mathrm{L}$ of CCK -8 reagent was added into each well and incubated for 4 hours at $37^{\circ} \mathrm{C}$. Absorbance at $450 \mathrm{~nm}$ was then measured by a microplate reader, and corresponding proliferation curves were plotted.

\section{Statistics}

All statistical analyses were performed using SPSS 20.0. The correlations between expression levels of Sirt3 and patients' characteristics were tested by chi-squared test. Survival analyses were conducted by Kaplan-Meier method and compared by log-rank test. Multivariate Cox regression analysis was used to identify independent prognostic factors. For the cellular experiments, data were presented as mean $\pm \mathrm{SD}$ from three independent experiments and compared with Student's $t$-test. $P<0.05$ was considered statistically significant.

\section{Results \\ Patient information}

Our retrospective cohort included 48 females and 66 males, with a median age of 51.0 years. Patients' clinical characteristics were evaluated by tumor size, WHO grade, and Karnofsky performance score. ${ }^{26}$ Briefly, 27, 45, and 42 glioma cases were classified as WHO II, WHO III, and WHO IV, respectively. In addition, the surgical strategy was retrieved of all the patients. Forty-eight patients underwent gross total resection, 35 patients underwent subtotal resection, and the other 31 patients underwent partial resection or biopsy. The median overall survival (OS) time was 31.0 months for all the enrolled patients, and the 5-year OS rate is $19.2 \%$. The detailed clinicopathological characteristics of this cohort are described in Table 1.

Table I The correlations between clinical parameters and Sirt3 expression in glioma patients

\begin{tabular}{|c|c|c|c|c|}
\hline \multirow[t]{2}{*}{ Variables } & \multirow{2}{*}{$\frac{\text { Patients }}{(n=1 \mid 4)}$} & \multicolumn{2}{|c|}{ Sirt3 expression } & \multirow[t]{2}{*}{$P$-value } \\
\hline & & $\begin{array}{l}\text { Low } \\
(n=60)\end{array}$ & $\begin{array}{l}\text { High } \\
(n=54)\end{array}$ & \\
\hline Gender & & & & 0.713 \\
\hline Female & 48 & 27 & $2 \mathrm{I}(43.8 \%)$ & \\
\hline Male & 66 & 33 & $33(50.0 \%)$ & \\
\hline Age (years) & & & & 0.238 \\
\hline$\leq 50$ & 55 & 27 & $28(50.9 \%)$ & \\
\hline$>50$ & 59 & 33 & $26(44.1 \%)$ & \\
\hline Tumor size & & & & 0.879 \\
\hline$\leq 5 \mathrm{~cm}$ & 69 & 47 & $22(31.9 \%)$ & \\
\hline$>5 \mathrm{~cm}$ & 45 & 13 & 32 (7I.1\%) & \\
\hline WHO grade & & & & $0.004 *$ \\
\hline II & 27 & 21 & $6(22.2 \%)$ & \\
\hline III & 45 & 21 & $24(53.3 \%)$ & \\
\hline IV & 42 & 18 & $24(57.1 \%)$ & \\
\hline Karnofsky score & & & & 0.379 \\
\hline$\leq 90$ & 69 & 39 & $30(43.5 \%)$ & \\
\hline$>90$ & 45 & 21 & $24(53.3 \%)$ & \\
\hline Surgery & & & & 0.122 \\
\hline GTR & 48 & 29 & 19 (39.6\%) & \\
\hline STR & 35 & 18 & 17 (48.6\%) & \\
\hline PR/biopsy & 31 & 13 & | 8 (58.1\%) & \\
\hline
\end{tabular}

Note: *Statistically significant.

Abbreviations: GTR, gross total resection; PR, partial resection; Sirt3, sirtuin 3; STR, subtotal resection. 


\section{Sirt3 is upregulated in glioma tissues}

The 114 tissue samples were used for evaluation of Sirt3 protein expression by IHC. According to the distinct staining patterns of Sirt3 (Figure 1A and B), we classified patients into low-expression group and high-expression group. The RT-qPCR results also showed that glioma tissues possessed higher Sirt3 mRNA levels than those of normal brain tissues (Figure $1 \mathrm{C}, P=0.038$ ). Importantly, by searching the The Cancer Genome Atlas database, we found that low mRNA transcription of Sirt3 indicates a better clinical outcome of glioma patients (Figure 1D, $P=0.0097$ ).

\section{Correlation between Sirt3 expression and clinicopathological factors in glioma patients}

As shown in Table 1, all 114 glioma patients were divided into high- or low-expression groups based on the median value of the IHC score in tumor tissues. According to chisquared test, Sirt3 expression was significantly correlated with the WHO grade $(P=0.004)$. Patients with more advanced tumor grade showed higher Sirt3 protein levels.

A

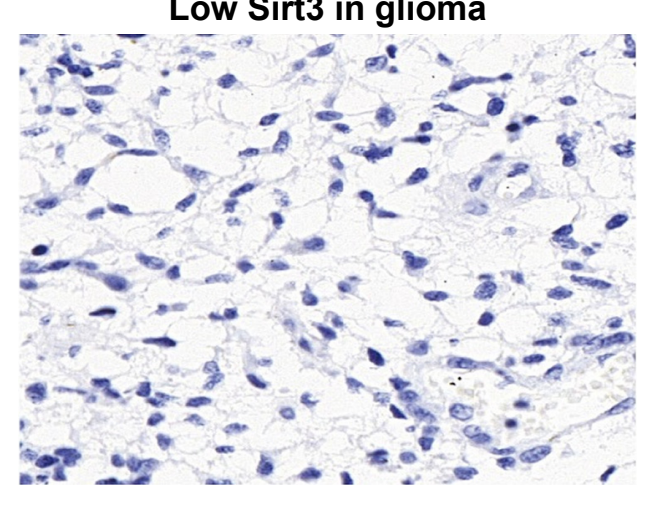

C

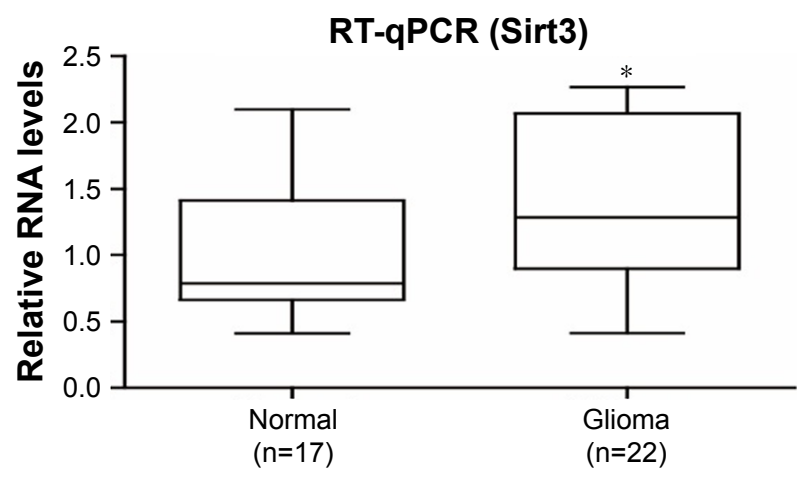

\section{Sirt3 is a novel biomarker indicating poor prognosis of glioma patients}

Because a higher Sirt3 was observed more frequently in glioma patients with advanced WHO grades, we hypothesized that it may help predict patients' clinical outcomes. We, therefore, analyzed the prognostic factors for the OS in glioma using Kaplan-Meier method (Figure 2, Table 2). A larger tumor size $(P=0.027)$, advanced WHO grades $(P=0.012)$, and noncurative surgical treatment $(P<0.001)$ were shown as unfavorable factors affecting OS. For example, the median OS for patients with WHO grades II, III, and IV was 49.0, 33.0, and 11.0 months, respectively (Table 2). Of note, patients with low Sirt3 expression showed a better prognosis than those with high Sirt3 expression (OS 39.3 \pm 2.9 months vs $25.0 \pm 3.1$ months, $P=0.003$ ).

Furthermore, we subjected the significant factors above into a Cox regression multivariate analysis model to explore the independent hazard effect of each factor (Table 3). Although tumor size showed no statistical significance, WHO grade (HR 1.927, 95\% CI 1.320-2.814, $P=0.001$ ), surgical treatment (HR 1.799, 95\% CI 1.164-2.781, $P=0.008$ ), and

B
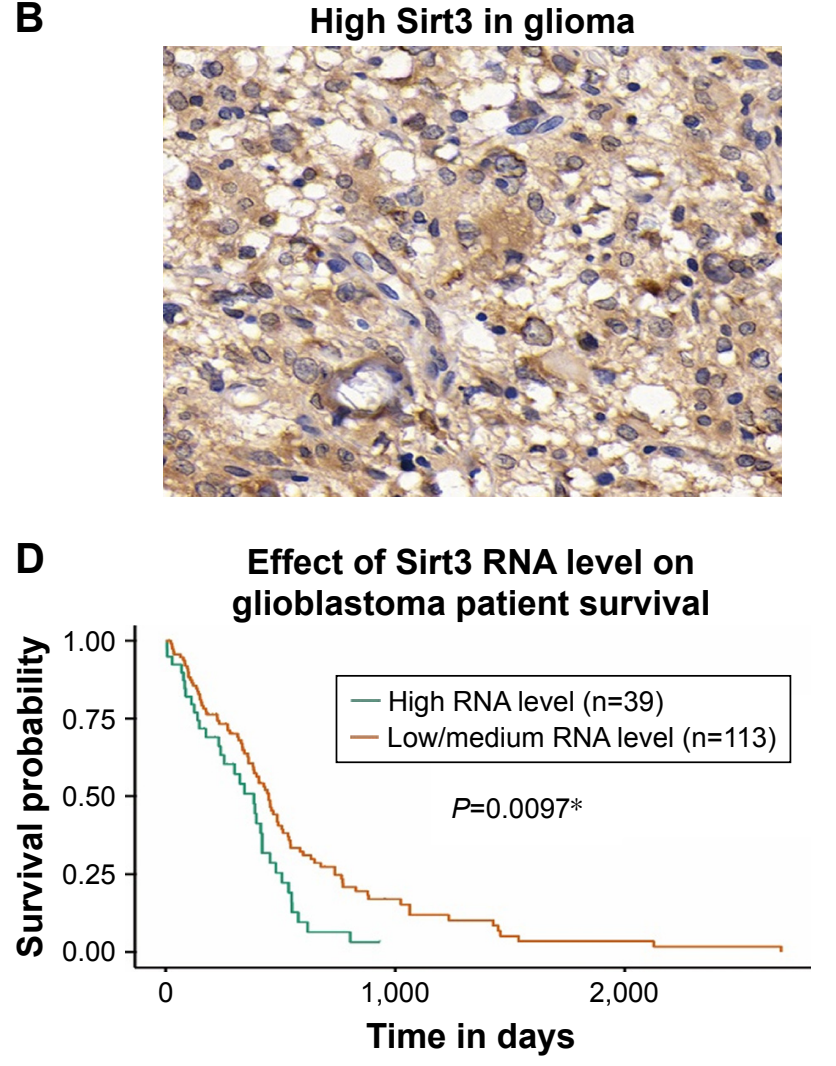

Figure I Sirt3 is upregulated in glioma tissues.

Notes: Representative low (A) and high (B) Sirt3 protein expression in clinical glioma tissues by IHC staining, showing the location of Sirt3 in cytoplasm. Magnification: 400X. (C) RT-qPCR results showed a significantly higher RNA level of Sirt3 in glioma tissues than that in normal brain tissues $(P=0.038)$. $* P<0.05$ by Student's $t$-test. (D) Higher Sirt3 RNA transcription indicated a poorer overall survival of glioma patients. $* P<0.05$ by log-rank test.

Abbreviations: IHC, immunohistochemistry; RT-qPCR, quantitative real-time PCR; Sirt3, sirtuin 3. 
A

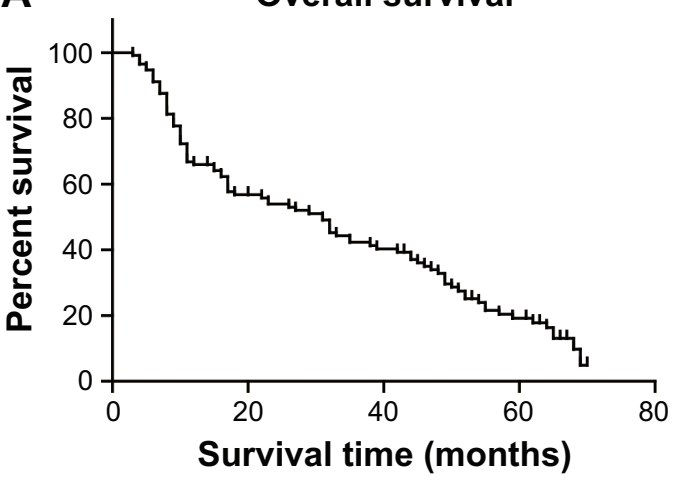

C

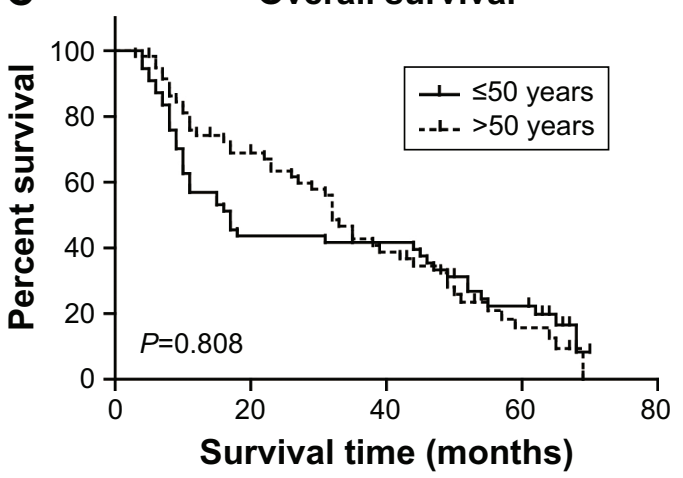

E
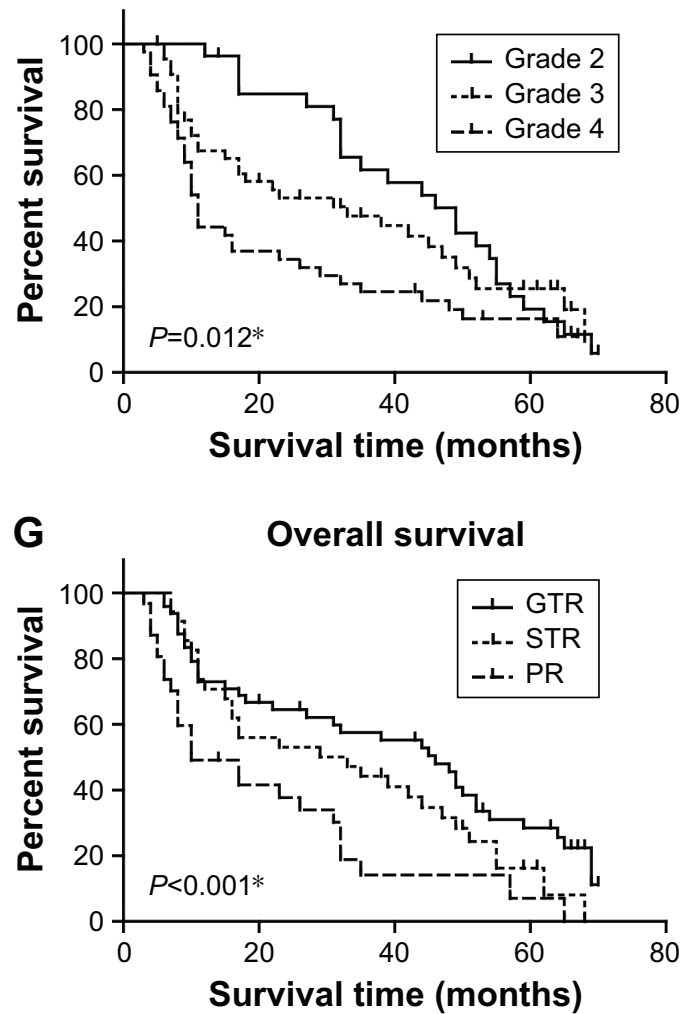

B Overall survival

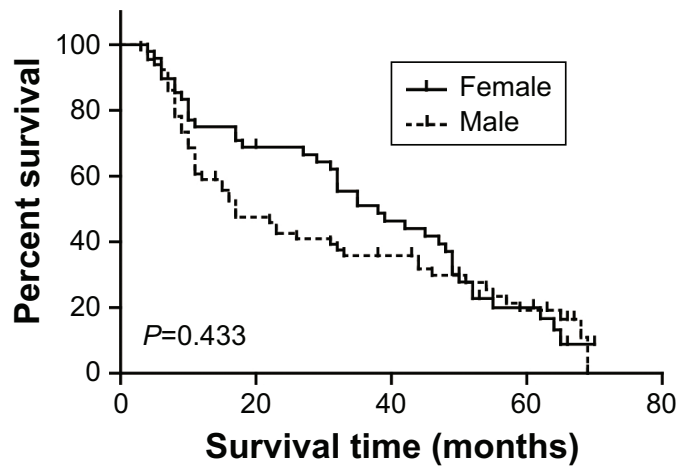

D Overall survival

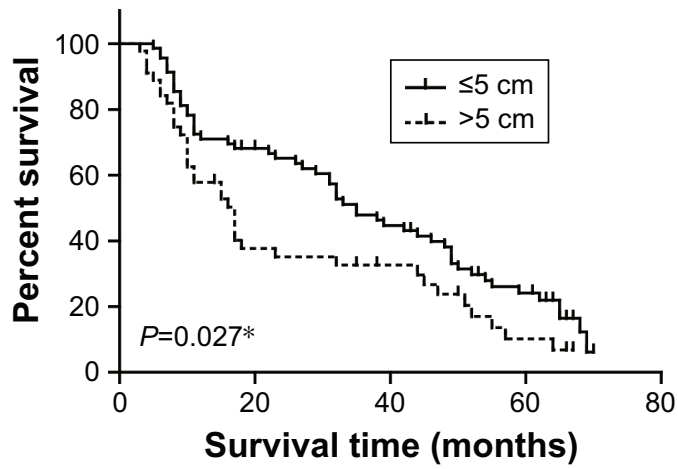

F Overall survival

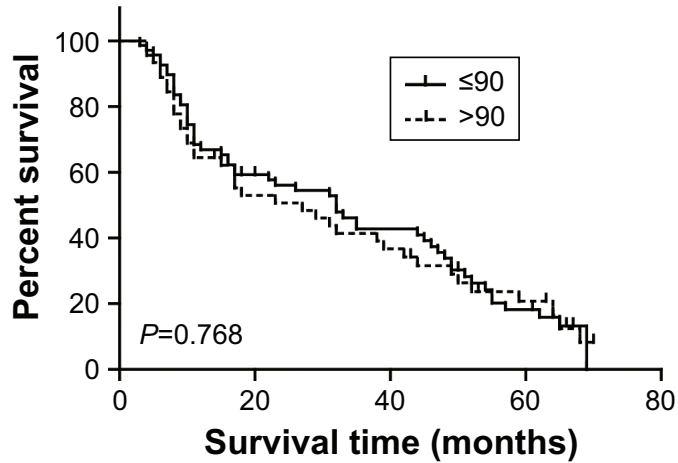

H Overall survival

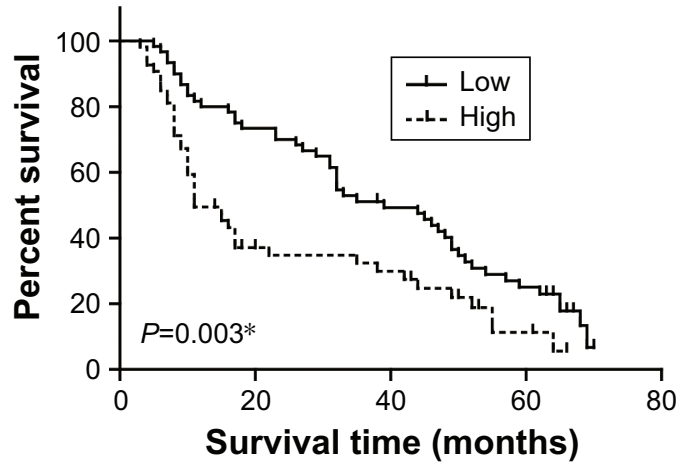

Figure 2 Analyses of the overall survival of glioma patients.

Notes: The overall survival curve for our enrolled cohort was showed by Kaplan-Meier plotting (A). Additionally, log-rank test was used to identify prognostic factors based on patients' gender (B), age (C), tumor size (D), WHO grade (E), Karnofsky score (F), surgical treatment $(\mathbf{G})$, and Sirt3 protein level $(\mathbf{H})$, respectively. $* P<0.05$.

Abbreviations: GTR, gross total resection; PR, partial resection; Sirt3, sirtuin 3; STR, subtotal resection. 
Table 2 Univariate analysis for overall survival of glioma patients

\begin{tabular}{|c|c|c|c|c|}
\hline \multirow[t]{2}{*}{ Variables } & \multirow{2}{*}{$\begin{array}{l}\text { Patients } \\
(n=I \mid 4)\end{array}$} & \multicolumn{2}{|c|}{$\begin{array}{l}\text { Overall survival time } \\
\text { (months) }\end{array}$} & \multirow[t]{2}{*}{$P$-value } \\
\hline & & Mean \pm SD & Median & \\
\hline Gender & & & & 0.433 \\
\hline Female & 48 & $36.7 \pm 3.2$ & 38.0 & \\
\hline Male & 66 & $30.0 \pm 3.1$ & 17.0 & \\
\hline Age (years) & & & & 0.808 \\
\hline$\leq 50$ & 55 & $30.8 \pm 3.5$ & 17.0 & \\
\hline$>50$ & 59 & $34.6 \pm 2.9$ & 32.0 & \\
\hline Tumor size & & & & $0.027^{*}$ \\
\hline$\leq 5 \mathrm{~cm}$ & 69 & $36.9 \pm 2.8$ & 35.0 & \\
\hline$>5 \mathrm{~cm}$ & 45 & $25.9 \pm 3.4$ & 17.0 & \\
\hline WHO grade & & & & $0.012^{*}$ \\
\hline II & 27 & $44.1 \pm 3.4$ & 49.0 & \\
\hline III & 45 & $34.6 \pm 3.8$ & 33.0 & \\
\hline IV & 42 & $23.7 \pm 3.5$ & 11.0 & \\
\hline Karnofsky score & & & & 0.768 \\
\hline$\leq 90$ & 69 & $33.7 \pm 2.9$ & 32.0 & \\
\hline$>90$ & 45 & $31.7 \pm 3.6$ & 27.0 & \\
\hline Surgery & & & & $<0.00 I^{*}$ \\
\hline GTR & 48 & $39.7 \pm 3.5$ & 46.0 & \\
\hline STR & 35 & $32.8 \pm 3.7$ & 33.0 & \\
\hline PR/biopsy & 31 & $21.6 \pm 3.7$ & 10.0 & \\
\hline Sirt3 expression & & & & $0.003^{*}$ \\
\hline Low & 60 & $39.3 \pm 2.9$ & 39.0 & \\
\hline High & 54 & $25.0 \pm 3.1$ & 11.0 & \\
\hline
\end{tabular}

Note: *Statistically significant.

Abbreviations: GTR, gross total resection; PR, partial resection; Sirt3, sirtuin 3; STR, subtotal resection.

Sirt3 expression (HR 1.602, 95\% CI 1.089-2.446, $P=0.017$ ) were all independently correlated with overall survival of glioma patients.

\section{Sirt3 enhances cell viability of glioma cells}

We next tested the functional mechanisms of Sirt3 in glioma. By analyzing its endogenous expression levels in different cell lines, we found that U87 and U251 glioma cells showed significantly higher Sirt3 levels than those in NHA cells (Figure 3A), which is consistent with the RT-qPCR results from clinical tissues samples.

By silencing or overexpressing Sirt3 in both U87 and U251 cells, we further identified that high Sirt3 expression

Table 3 Multivariate analysis for the prognostic factors of glioma patients

\begin{tabular}{llll}
\hline Variables & HR & $\mathbf{9 5 \% ~ C l}$ & P-value \\
\hline Tumor size & 1.105 & $0.702-1.739$ & 0.666 \\
WHO grade & 1.927 & $1.320-2.814$ & $0.00 I^{*}$ \\
Surgery & 1.799 & $1.164-2.781$ & $0.008^{*}$ \\
Sirt3 expression & 1.602 & $1.089-2.446$ & $0.017^{*}$ \\
\hline
\end{tabular}

Note: *Statistically significant.

Abbreviation: Sirt3, sirtuin 3. can significantly upregulate the tumor cell viability, as revealed by the CCK-8 assay (Figure 3B and C).

\section{Sirt3 interacts with Ku70 and regulates its acetylation level}

There had been evidence that Sirt3 may regulate the function of Ku70 protein in cardiomyocytes during response to oxidative stress. ${ }^{27} \mathrm{Ku} 70$ plays critical roles in controlling the cellular localization of BAX, the mitochondria translocation of which will induce cell apoptosis. ${ }^{28}$ Moreover, the interaction between $\mathrm{Ku} 70$ and BAX was acetylation dependent; once Ku70 is deacetylated, it will release BAX and allow it to transport into mitochondria. Therefore, we performed immunoprecipitation assay to test the existence of Sirt3-Ku70 signaling in U87 glioma cells. As expected, Ku70 was successfully pulled down by immunoprecipitating Sirt3 (Figure 3D). In addition, the acetylation level of Ku70 was negatively correlated with the Sirt3 level (Figure 3E), indicating that Ku70 may be a novel substrate of Sirt3 in glioma cells. Finally, we verified that silencing Sirt3 can significantly increase the BAX level in mitochondria, which subsequently leads to cytochrome c release and caspase 3 cleavage (Figure 3F). In contrast, overexpressing Sirt3 showed completely opposite effects.

\section{Sirt3 may promote tumor progression by targeting Ku70}

To further investigate the role of Ku70 in Sirt3 signaling pathway, we next silenced Ku70 in U87 cells that were stably expressing Sirt3. Accordingly, we found that Ku70 siRNA significantly decreased the cell viability (Figure 4A). In addition, the levels of mitochondria BAX and cytosol cytochrome c were enhanced by silencing Ku70, which attenuated the tumor-promoting effects of Sirt3 overexpression (Figure 4B). Thus, we concluded that Sirt3 can promote glioma progression at least partially by Ku70 pathway.

\section{Discussion}

Recently, novel strategies on identifying tumor biomarkers are developing rapidly such as the noninvasive platelet screening. ${ }^{29}$ Another trend is that more and more newly identified biomarkers possess enzymatic functions, especially the phosphorylation, ubiquitination, and acetylation. The protein acetylation mainly occurs on either $\mathrm{N}$ terminus or lysine residues. Acetylation is highly dynamic, and its modification level is balanced by acetyltransferases and deacetylases.

A good example on how acetylation regulates tumor progression is the Ku70-BAX signaling pathway. Briefly, the 
A

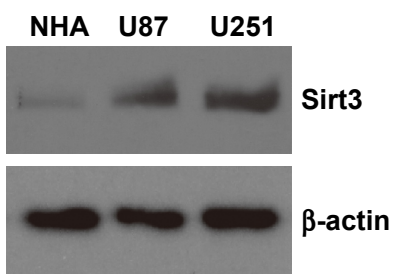

B

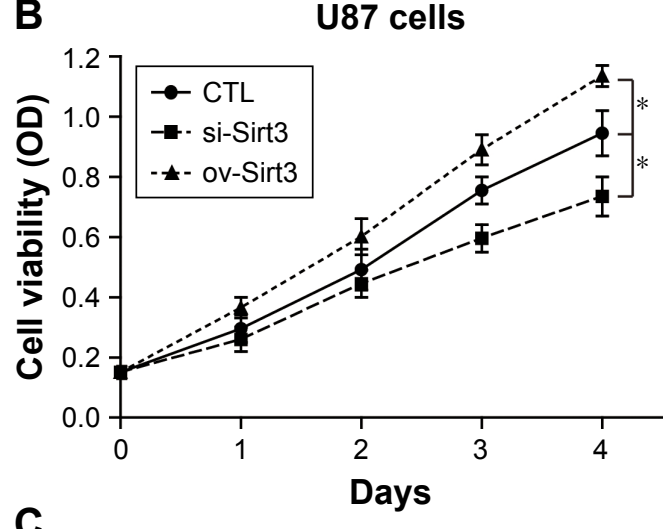

C

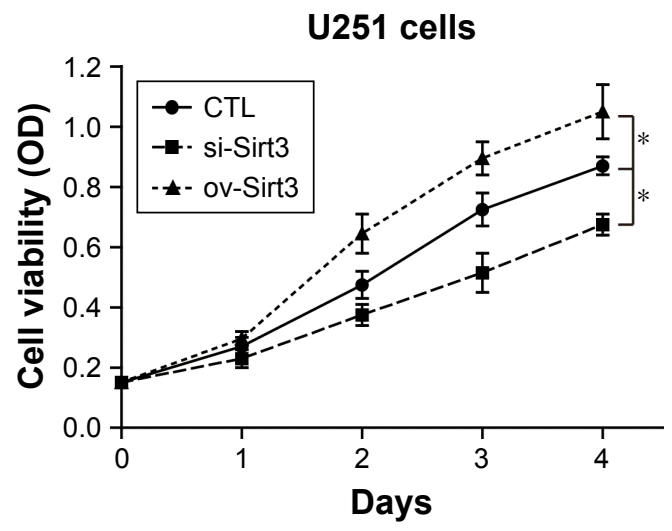

D

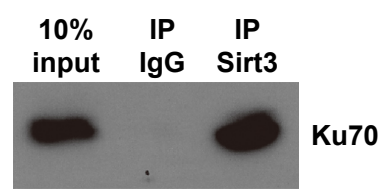

E

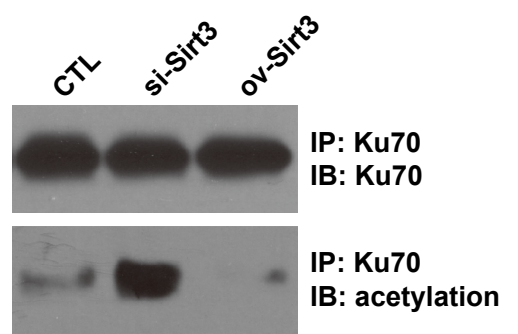

$\mathbf{F}$
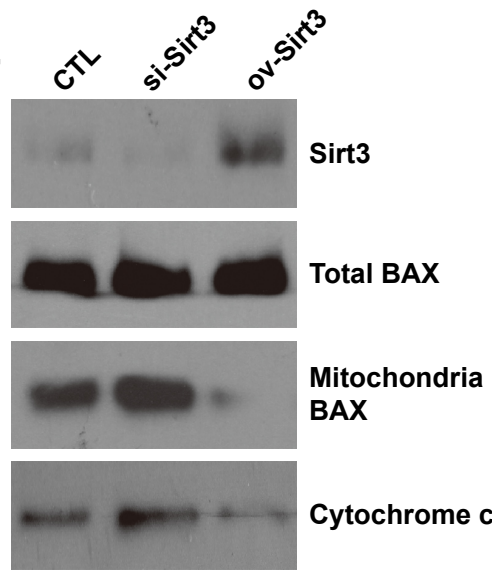

Cytochrome c

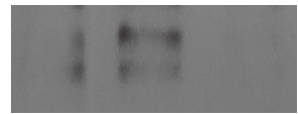

Cleaved

caspase 3

\section{$\beta$-actin}

Figure 3 Sirt3 enhances glioma cell viability.

Notes: (A) The protein expression level of Sirt3 was significantly higher in U87 and U25I glioma cell lines than that in normal human astrocytes (NHA cells). Overexpressing Sirt3 enhanced growth viability of U87 (B) and U25I cells (C), while silencing Sirt3 showed opposite effects. (D) Immunoprecipitation experiments demonstrated the interaction between Sirt3 and Ku70 proteins. (E) Sirt3 negatively regulated the acetylation of Ku70 without affecting its total protein level. (F) Silencing Sirt3 upregulated the BAX protein translocation into mitochondria and promoted the release of cytochrome $\mathrm{c}$ and cleavage of caspase 3 . On the other hand, overexpressing Sirt3 showed opposite effects on the apoptotic proteins. All experiments were performed in triplicate and repeated for three times. $* P<0.05$ compared to control (CTL) cells by Student's $t$-test. Abbreviations: BAX, Bcl-2-associated X protein; IB, immunoblotting; IP, immunoprecipitation; NHA, normal human astrocytes; ov, overexpression; si, siRNA; Sirt3, sirtuin 3.

BAX-mediated apoptosis can be suppressed by overexpression of Ku70 in mammalian cells, but enhanced by downregulation of Ku70. ${ }^{30}$ Following studies demonstrated that $\mathrm{Ku} 70$ can directly interact with BAX in cytosol, thus preventing the apoptotic translocation of BAX to mitochondria. It is interesting that the nonacetylation status of $\mathrm{Ku} 70$ is a non-negligible prerequisite for the stable interaction between $\mathrm{Ku} 70$ and BAX. Under certain apoptotic conditions, the $\mathrm{Ku} 70$ is acetylated by $\mathrm{BAX}$, subsequently releases BAX, allows it to transport into mitochondria, and finally induces cell apoptosis. Therefore, it is reasonable that disruption of $\mathrm{Ku} 70-\mathrm{BAX}$ interaction by acetylating $\mathrm{Ku} 70$ may lead to tumor cell apoptosis. Indeed, overexpression of CREB-binding protein, a known acetyltransferase toward $\mathrm{Ku} 70$, can induce the apoptosis of HeLa cells. ${ }^{31}$ Consistently, pharmacological inhibiting or silencing $\mathrm{Ku} 70$ deacetylase, such as histone deacetylase 6, also successfully triggered BAX-dependent apoptosis of neuroblastoma cells. ${ }^{32}$ The tumor-related significance of Ku70-BAX 
A

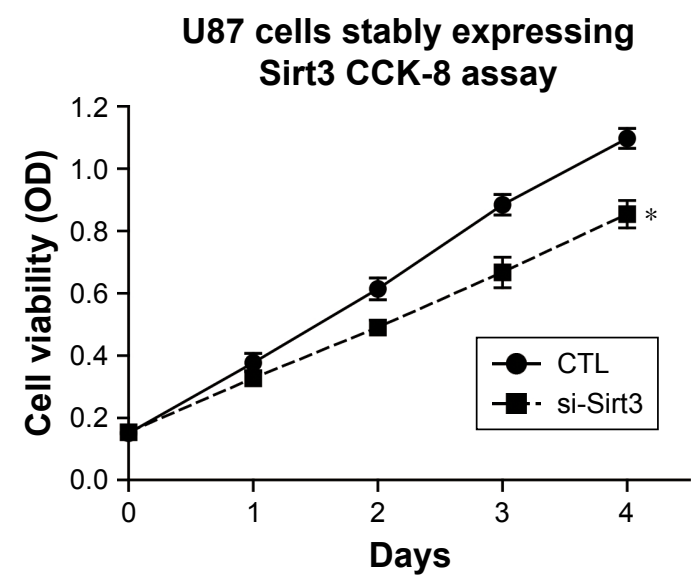

B

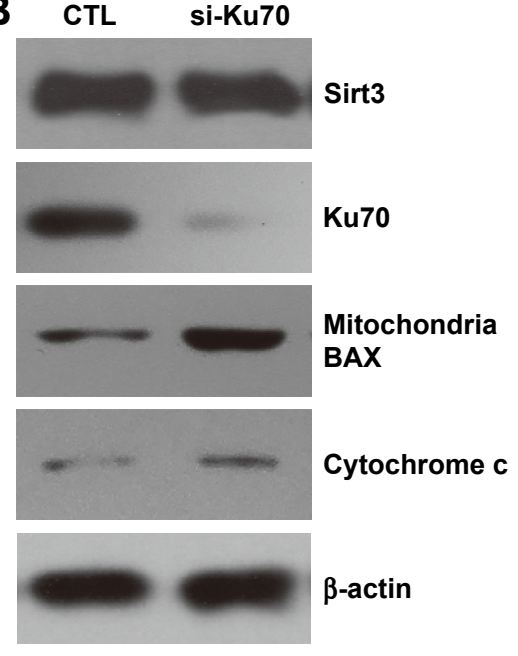

Figure 4 Silencing Ku70 attenuates the pro-proliferative effects of Sirt3 in glioma cells.

Notes: (A) CCK-8 assay showed that Ku70 siRNA significantly inhibited cell proliferation of U87 cells that were stably expressing Sirt3. (B) Treatment by Ku70 siRNA enhanced BAX protein translocation into mitochondria and promoted the release of cytochrome $\mathrm{c}$.

Abbreviations: BAX, Bcl-2-associated X protein; CTL, control; si, siRNA.

interaction had also been revealed in prostate cancer, colon cancer, and osteosarcoma. ${ }^{28,33,34}$

On the other hand, overexpression of $\mathrm{Ku} 70$ upstream deacetylases may exert tumor-promoting functions by inhibiting cell apoptosis. Here we first showed that Sirt3, a member of the Sirt protein family that catalyzes deacetylation, was highly expressed in glioma tissues compared with normal brain tissues. Survival analyses revealed an independent prognostic effect of higher Sirt3 expression on predicating poor prognosis. We then performed cellular experiments to see whether Sirt3 can directly regulate glioma cell viability. As expected, overexpression of Sirt 3 enhanced cell growth, while silencing Sirt3 via siRNA significantly promoted cell apoptosis. Furthermore, our data demonstrated the involvement of Ku70 deacetylation in Sirt3 signaling pathways, which was consistent with its enzymatic relationship in cardiomyocytes. ${ }^{27}$ Consistently, a recent study reported that treatment by petunidin-3-O-glucoside (Pt3glc) displayed potent antiproliferative effects and also decreased the Sirt3 protein level. ${ }^{35}$ Further investigation is needed to determine whether Pt3glc functions by directly targeting Sirt3. Therefore, targeting the expression of Sirt3 may be another novel therapeutic direction for aging-associated tumors. ${ }^{36}$ Finally, we verified that Sirt3 overexpression inhibited glioma cell apoptosis by stabilizing $\mathrm{Ku} 70-\mathrm{BAX}$ interaction in cytosol.

Our study therefore provided evidence of the potential function of Sirt3 in glioma progression. Since several high throughput strategies are being used to screen Sirt3 inhibitors, it is highly likely that Sirt3 will be a novel therapeutic drug target in the near future. ${ }^{37-39}$ Moreover, several groups are focusing on developing drugs targeting the Ku70-BAX interaction, ${ }^{33,40}$ which may be combined with Sirt3 inhibitors in treating malignancies. A similar example is the interaction between Sirt3 and superoxide dismutase 2 (SOD2) protein. Cheng et al reported that inhibiting the Sirt3 expression or disrupting Sirt3-SOD2 interaction may help attenuate the drug resistance of linalool, a specific drug targeting SOD2, on glioma cells. ${ }^{41}$

\section{Conclusion}

In summary, our study revealed the expression patterns of Sirt3 in glioma patients for the first time and demonstrated its correlations with the disease stages and prognosis. Identification of Sirt3 as a novel biomarker for glioma and revealing its functional mechanisms would be invaluable for treatment improvement.

\section{Disclosure}

The authors report no conflicts of interest in this work.

\section{References}

1. Deangelis LM. Brain tumors. N Engl J Med. 2001;344(2):114-123.

2. Gao J, Ciriello G, Sander C, Schultz N. Collection, integration and analysis of cancer genomic profiles: from data to insight. Curr Opin Genet Dev. 2014;24:92-98.

3. Liu L, Li W, Geng S, et al. Slit2 and Robo1 expression as biomarkers for assessing prognosis in brain glioma patients. Surg Oncol. 2016; 25(4):405-410.

4. Siegel RL, Miller KD, Jemal A. Cancer Statistics. CA Cancer J Clin. 2018;68(1):7-30.

5. Li X, Zheng J, Diao H, Liu Y. RUNX3 is down-regulated in glioma by Myc-regulated miR-4295. J Cell Mol Med. 2016;20(3):518-525. 
6. Osborne B, Bentley NL, Montgomery MK, Turner N. The role of mitochondrial sirtuins in health and disease. Free Radic Biol Med. 2016;100:164-174.

7. Winnik S, Auwerx J, Sinclair DA, Matter CM. Protective effects of sirtuins in cardiovascular diseases: from bench to bedside. Eur Heart J. 2015;36(48):3404-3412.

8. Yu W, Dittenhafer-Reed KE, Denu JM. SIRT3 protein deacetylates isocitrate dehydrogenase 2 (IDH2) and regulates mitochondrial redox status. J Biol Chem. 2012;287(17):14078-14086.

9. Scher MB, Vaquero A, Reinberg D. SirT3 is a nuclear NAD+-dependent histone deacetylase that translocates to the mitochondria upon cellular stress. Genes Dev. 2007;21(8):920-928.

10. Koentges C, Pfeil K, Meyer-Steenbuck M, et al. Preserved recovery of cardiac function following ischemia-reperfusion in mice lacking SIRT3. Can J Physiol Pharmacol. 2016;94(1):72-80.

11. Torrens-Mas M, Oliver J, Roca P, Sastre-Serra J. SIRT3: oncogene and tumor suppressor in cancer. Cancers. 2017;9(12):90.

12. Desouki MM, Doubinskaia I, Gius D, Abdulkadir SA. Decreased mitochondrial SIRT3 expression is a potential molecular biomarker associated with poor outcome in breast cancer. Hum Pathol. 2014; 45(5):1071-1077.

13. Liu Y, Liu YL, Cheng W, Yin XM, Jiang B. The expression of SIRT3 in primary hepatocellular carcinoma and the mechanism of its tumor suppressing effects. Eur Rev Med Pharmacol Sci. 2017;21(5):978-998.

14. Yu W, Denu RA, Krautkramer KA, et al. Loss of SIRT3 provides growth advantage for B cell malignancies. J Biol Chem. 2016; 291(7):3268-3279.

15. Liu C, Huang Z, Jiang H, Shi F. The sirtuin 3 expression profile is associated with pathological and clinical outcomes in colon cancer patients. Biomed Res Int. 2014;2014:1-9.

16. Cui Y, Qin L, Wu J, et al. SIRT3 enhances glycolysis and proliferation in SIRT3-expressing gastric cancer cells. PLoS One. 2015;10(6): e0129834.

17. George J, Nihal M, Singh CK, Zhong W, Liu X, Ahmad N. Proproliferative function of mitochondrial sirtuin deacetylase SIRT3 in human melanoma. J Invest Dermatol. 2016;136(4):809-818.

18. Choi J, Koh E, Lee YS, et al. Mitochondrial Sirt3 supports cell proliferation by regulating glutamine-dependent oxidation in renal cell carcinoma. Biochem Biophys Res Commun. 2016;474(3):547-553.

19. Zhang Q, Fan H, Zou Q, et al. TEAD4 exerts pro-metastatic effects and is negatively regulated by miR6839-3p in lung adenocarcinoma progression. J Cell Mol Med. 2018;22(7):3560-3571.

20. Liu H, Xu Y, Zhang Q, et al. Correlations between TBL1XR1 and recurrence of colorectal cancer. Sci Rep. 2017;7:44275

21. Xu Y, Yang X, Li Z, et al. Sprouty2 correlates with favorable prognosis of gastric adenocarcinoma via suppressing FGFR2-induced ERK phosphorylation and cancer progression. Oncotarget. 2017;8(3):4888.

22. Tan W, Pan M, Liu H, Tian H, Ye Q, Liu H. Ergosterol peroxide inhibits ovarian cancer cell growth through multiple pathways. Onco Targets Ther. 2017;10:3467-3474.

23. Liu H, Zhang Q, Li K, et al. Prognostic significance of USP33 in advanced colorectal cancer patients: new insights into $\beta$-arrestindependent ERK signaling. Oncotarget. 2016;7(49):81223-81240.

24. Fly R, Lloyd J, Krueger S, Fernie A, van der Merwe MJ. Improvements to define mitochondrial metabolomics using nonaqueous fractionation. Methods Mol Biol. 2015;1305:197-210.
25. Hou S, du P, Wang P, Wang C, Liu P, Liu H. Significance of MNK1 in prognostic prediction and chemotherapy development of epithelial ovarian cancer. Clin Transl Oncol. 2017;19(9):1107-1116.

26. Johnson MJ, Bland JM, Davidson PM, et al. The relationship between two performance scales: New York Heart Association Classification and Karnofsky Performance Status Scale. J Pain Symptom Manage. 2014;47(3):652-658.

27. Sundaresan NR, Samant SA, Pillai VB, Rajamohan SB, Gupta MP. SIRT3 is a stress-responsive deacetylase in cardiomyocytes that protects cells from stress-mediated cell death by deacetylation of $\mathrm{Ku} 70$. Mol Cell Biol. 2008;28(20):6384-6401.

28. Trougakos IP, Lourda M, Antonelou MH, et al. Intracellular clusterin inhibits mitochondrial apoptosis by suppressing p53-activating stress signals and stabilizing the cytosolic Ku70-Bax protein complex. Clin Cancer Res. 2009;15(1):48-59.

29. Zhang Q, Liu H, Zhu Q, et al. Patterns and functional implications of platelets upon tumor “education." Int J Biochem Cell Biol. 2017;90: 68-80.

30. Sawada M, Sun W, Hayes P, Leskov K, Boothman DA, Matsuyama S. $\mathrm{Ku} 70$ suppresses the apoptotic translocation of Bax to mitochondria. Nat Cell Biol. 2003;5(4):320-329.

31. Cohen HY, Lavu S, Bitterman KJ, et al. Acetylation of the $\mathrm{C}$ terminus of Ku70 by CBP and PCAF controls Bax-mediated apoptosis. Mol Cell. 2004;13(5):627-638.

32. Subramanian C, Jarzembowski JA, Opipari AW, Castle VP, Kwok RP. HDAC6 deacetylates $\mathrm{Ku} 70$ and regulates $\mathrm{Ku} 70$-Bax binding in neuroblastoma. Neoplasia. 2011;13(8):726-734.

33. Shukla S, Fu P, Gupta S. Apigenin induces apoptosis by targeting inhibitor of apoptosis proteins and $\mathrm{Ku} 70$-Bax interaction in prostate cancer. Apoptosis. 2014;19(5):883-894.

34. Pucci S, Mazzarelli P, Paola M, Sesti F, et al. Interleukin-6 affects cell death escaping mechanisms acting on Bax-Ku70-Clusterin interactions in human colon cancer progression. Cell Cycle. 2009;8(3):473-481.

35. Wang G, Fu XL, Wang JJ, Guan R, Sun Y, To ST. Inhibition of glycolytic metabolism in glioblastoma cells by Pt3glc combinated with PI3K inhibitor via SIRT3-mediated mitochondrial and PI3K/Akt-MAPK pathway. J Cell Physiol. Epub 16 Jan 2018.

36. Son MJ, Ryu JS, Kim JY, et al. Upregulation of mitochondrial NAD ${ }^{+}$ levels impairs the clonogenicity of SSEA $1^{+}$glioblastoma tumorinitiating cells. Exp Mol Med. 2017;49(6):e344.

37. Salo HS, Laitinen T, Poso A, Jarho E, Lahtela-Kakkonen M. Identification of novel SIRT3 inhibitor scaffolds by virtual screening. Bioorg Med Chem Lett. 2013;23(10):2990-2995.

38. Disch JS, Evindar G, Chiu CH, et al. Discovery of thieno[3,2-d] pyrimidine-6-carboxamides as potent inhibitors of SIRT1, SIRT2, and SIRT3. J Med Chem. 2013;56(9):3666-3679.

39. Chen Y, Fu LL, Wen X, et al. Sirtuin-3 (SIRT3), a therapeutic target with oncogenic and tumor-suppressive function in cancer. Cell Death Dis. 2014;5(2):e1047.

40. Sawada M, Hayes P, Matsuyama S. Cytoprotective membrane-permeable peptides designed from the Bax-binding domain of Ku70. Nat Cell Biol. 2003;5(4):352-357.

41. Cheng Y, Dai C, Zhang J. SIRT3-SOD2-ROS pathway is involved in linalool-induced glioma cell apoptotic death. Acta Biochim Pol. 2017; 64(2):343-350.
OncoTargets and Therapy

\section{Publish your work in this journal}

OncoTargets and Therapy is an international, peer-reviewed, open access journal focusing on the pathological basis of all cancers, potential targets for therapy and treatment protocols employed to improve the management of cancer patients. The journal also focuses on the impact of management programs and new therapeutic agents and protocols on

\section{Dovepress}

patient perspectives such as quality of life, adherence and satisfaction. The manuscript management system is completely online and includes a very quick and fair peer-review system, which is all easy to use. Visit http://www.dovepress.com/testimonials.php to read real quotes from published authors. 